Wilfrid Laurier University

Scholars Commons @ Laurier

Physics and Computer Science Faculty

Publications

Physics and Computer Science

1992

\title{
Transient Chaos in a Parametrically Damped Pendulum
}

James A. Blackburn

Wilfrid Laurier University, jabjabjab@cogeco.ca

H.J.T. Smith

University of Waterloo

Darran E. Edmundson

Simon Fraser University

Follow this and additional works at: https://scholars.wlu.ca/phys_faculty

\section{Recommended Citation}

Blackburn, James A.; Smith, H.J.T.; and Edmundson, Darran E., "Transient Chaos in a Parametrically Damped Pendulum" (1992). Physics and Computer Science Faculty Publications. 55.

https://scholars.wlu.ca/phys_faculty/55

This Article is brought to you for free and open access by the Physics and Computer Science at Scholars Commons @ Laurier. It has been accepted for inclusion in Physics and Computer Science Faculty Publications by an authorized administrator of Scholars Commons @ Laurier. For more information, please contact scholarscommons@wlu.ca. 


\title{
Transient chaos in a parametrically damped pendulum
}

\author{
James A. Blackburn \\ Department of Physics and Computing, Wilfrid Laurier University, Waterloo, Ontario, Canada N2L $3 C 5$ \\ H. J. T. Smith \\ Department of Physics, University of Waterloo, Waterloo, Ontario, Canada N2L 3G1 \\ Darran E. Edmundson \\ Department of Physics, Simon Fraser University, Burnaby, British Columbia, Canada V5A IS6
}

(Received 18 March 1991; revised manuscript received 30 August 1991)

\begin{abstract}
The parametrically damped pendulum exhibits chaotic transients over a sizable portion of its state space. Using the techniques of interpolated cell mapping (ICM), it has been found that these transients arise from the spatial congruence of the basin of attraction of a stable stationary solution and the remnant of a destroyed strange attractor. The mean transient lifetime is found to scale as $\left|\epsilon-\epsilon_{c}\right|^{-\gamma}$, where $\epsilon$ is a system control parameter, $\epsilon_{c}$ is its value at the chaotic boundary, and $\gamma$ is the critical exponent. Also included are ICM data that reveal the nature of basin transformations as nonchaotic boundaries in the state diagram are traversed.
\end{abstract}

PACS number(s): 05.45.+b

\section{INTRODUCTION}

If a nonlinear system is exhibiting chaotic motion and this behavior is monitored over a sufficient period of time, one of a number of possible scenarios might be observed. In the first, chaos might simply persist for as long as the system is observed. In some cases, the chaotic motion might abruptly terminate, only to resume after some "laminar time." Such repetitive bursts of chaos are known as intermittency $[1,2]$. The mean laminar time is known to scale as $\left|g-g_{c}\right|^{-\gamma}$ where $g$ is a system control parameter and $g_{c}$ is its critical value. A considerable literature now exists on this topic; we mention just two studies by way of example. For a driven damped pendulum, Gwinn and Westervelt [3] observed switching between metastable running modes and found that the average time spent in either of these modes was described by a scaling exponent $\gamma=0.5 \pm 0.15$. Intermittency induced by an interior crisis in an experimental system comprised of a parametrically driven magnetoelastic ribbon yielded [4] a scaling exponent $\gamma=0.086 \pm 0.03$.

A third scenario occurs when, after some length of time, chaos permanently ceases, and the system then continues in some form of multiperiodic motion. Grebogi, Ott, and Yorke [5] showed that when such transient chaos is associated with a boundary crisis, then the mean transient time $T$ scales as $\left|g-g_{c}\right|^{-\gamma}$. In this paper we describe numerical simulations carried out on a parametrically damped pendulum, and show that the transient chaos which arises also follows the scaling law $\left|g-g_{c}\right|^{-\gamma}$, but is not associated with a boundary crisis.

Much of the discussion is concerned with basins of attraction: sets of initial conditions $(\theta, d \theta / d t)$ for which a system ultimately arrives at a specified attractor. The most direct way of determining a basin is to numerically integrate the differential equation for each possible starting point and record those values which eventually lead to the attractor. This approach has been used to generate high-resolution basins containing as many as one million initial conditions [6-8]. Of course the computational demands are formidable and generally require the use of a supercomputer. A computationally efficient algorithm was developed by Hsu [9], and Hsu and Guttalu [10] to perform cell-to-cell mappings for dynamical systems and to extract from the mappings equilibrium states, periodic motions, and their domains of attraction. Cell mapping has been employed to study various aspects of rf-driven Josephson junctions [11,12], including the appearance of both interior and boundary crises [13].

A more recent development due to Tongue $[14,15]$ is known as interpolated cell mapping (ICM). ICM reduces a continuous time system to a discrete mapping as follows. An $M \times N$ rectangular mesh is placed over the area of phase space containing the attractors of interest. Each cell in the mesh is then distorted by a Poincare mapping of its vertices. Thus the Poincaré iterate of an arbitrary initial condition (belonging to a particular cell in the mesh) can be approximated by linearly interpolating within the distorted cell. The advantage of ICM over other cell mapping techniques is that one can generate an infinite number of distinct orbits from an $M \times N$ mesh. Tongue demonstrated the method of applying it to the determination of a basin of attraction for the forced damped pendulum, and showed that a grid of $101 \times 101$ could generate a basin of equivalent resolution to one obtained from a direct computation involving $1001 \times 1001$ starting points.

\section{PHYSICAL SYSTEM AND NUMERICAL METHOD}

In a previous study [16], we described experiments and numerical simulations which were carried out on a parametrically damped pendulum. The equation governing this system is 


$$
I \frac{d^{2} \theta}{d t^{2}}+[A+B \sin (\omega t)] \frac{d \theta}{d t}+m g b \sin (\theta)=0 .
$$

The pendulum consists of a mass $m$ situated a distance $b$ from the rotation axis; $I$ is the total moment of inertia of all rotating components of the apparatus. The key parameters were shown to be $\omega_{0}=(m g b / I)^{-1 / 2}, \Omega=\omega / \omega_{0}$, $Q=(m g b I)^{1 / 2} / A$, and $\epsilon=B / A$. Throughout this work, $Q$ will be set at 18.33 , the value determined for the original experimental apparatus. With time normalized to $\omega_{0}^{-1}$, Eq. (1) becomes

$$
\frac{d^{2} \theta}{d t^{* 2}}+Q^{-1}\left[1+\epsilon \sin \left(\Omega t^{*}\right)\right] \frac{d \theta}{d t^{*}}+\sin (\theta)=0 .
$$

An important property of this equation is readily apparent: it is always satisfied by $d \theta / d t=\theta=0$ (such is not the case for the conventional torque driven pendulum). However, it should be noted that this particular solution is not always stable against small perturbations. The state diagram determined in that previous study is reproduced here as Fig. 1. In the present work, we are particularly interested in the region to the left of the $V$-shaped domain. Within this region, chaotic transients are common, and in addition, the stationary state is stable. Extensive use of the interpolated cell mapping algorithm has been made in the present work. The first stage of any computation was to generate the mapping function from a $101 \times 101$ grid in phase space using a fourth-order Runge-Kutta integration routine. The integration time step was set at one-hundredth of the drive period. For each new $\epsilon$ and/or $\Omega$, a new mapping function was evaluated. To determine a basin of attraction, a small target area around the attractor was first specified. Points were then selected from a $371 \times 357$ grid (these dimensions were set by the maximum number of pixels which would fill the designated graphic area on a VGA monitor), and for each one the mapping function was employed to follow the evolution of the initial point after each successive period of the drive term. If, within some specified maximum time allowance, the phase point fell inside the target and remained there for several more cycles, then that initial point was labeled as a member of the basin of attraction.

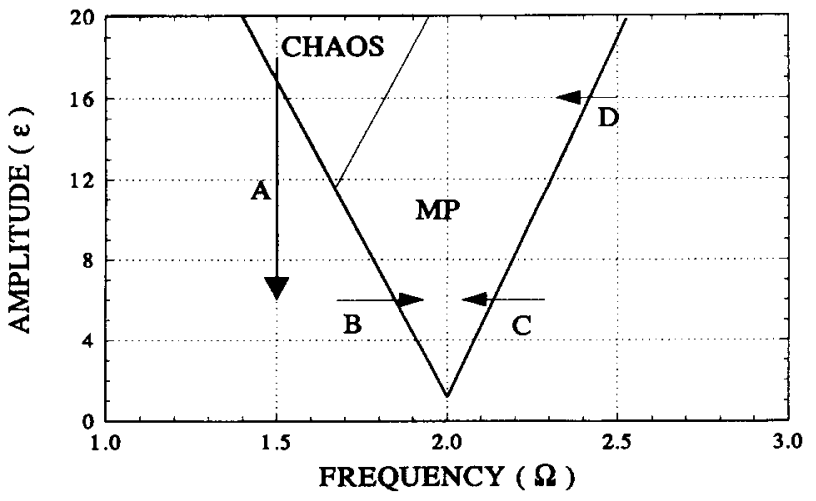

FIG. 1. State diagram for the parametrically damped pendulum. MP denotes a region in which the pendulum motion is periodic or multiperiodic. (a)

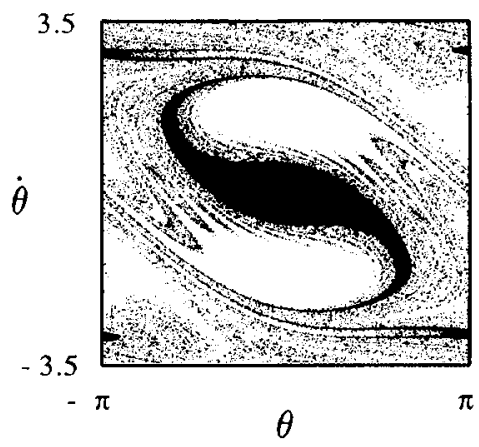

(b)

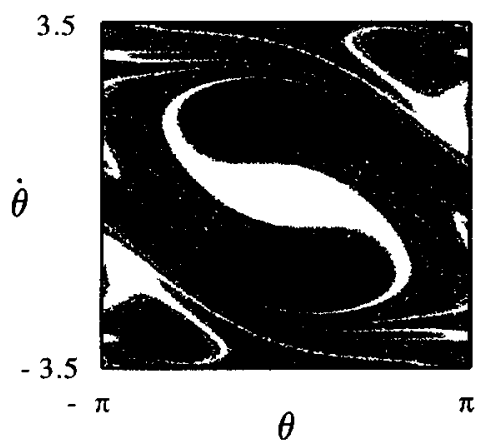

FIG. 2. (a) Basin of attraction (in black) for the stationary solution of the pendulum with $\epsilon=6.0$ and $\Omega=1.70$. (b) Basin of attraction of the oscillating solution.

As an illustration of ICM, consider the parameter choice: $\epsilon=6.0, \Omega=1.70$. This case was considered in Ref. 16, where it was shown that there are four coexisting attractors (a) the stationary state $\theta=d \theta / d t=0$; (b) an oscillating solution; (c) a clockwise running solution; (d) a counterclockwise running solution. Using interpolated cell mapping, we obtained the basin of attraction shown in Fig. 2(a) for the stationary solution, and the basin shown in Fig. 2(b) for the oscillating solution. A comparison of Fig. 2(a) with Fig. 6 of Ref. [16] confirms the high-resolution achievable with ICM. Each basin determination with ICM required about $3 \mathrm{~h}$ on a Compaq $486 / 25$ desktop computer, a speed increase of about 30 over the "brute force" approach used previously.

\section{STRANGE ATTRACTOR REMNANTS AND STABLE BASINS}

In this section we consider states of the system located along scan line $A$ in Fig. 1. At this particular $\Omega(1.50)$, the critical value of $\epsilon$ is $\epsilon_{c}=17.2$; above $\epsilon_{c}$ the stationary state is unstable and the motion is permanently chaotic. A typical strange attractor for this domain is shown in Fig. 3.

As noted earlier, below the chaotic boundary at $\epsilon_{c}$, the stationary state $\theta=d \theta / d t=0$ becomes stable; hence crossing the critical line from above leads to the birth of a new attractor (the origin) and its associated basin of attraction. The former strange attractor which existed just 


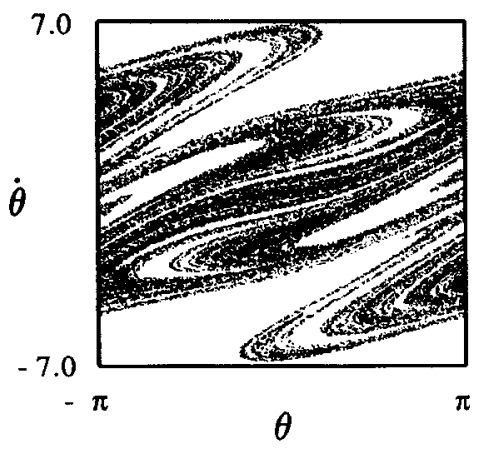

FIG. 3. Strange attractor for $\epsilon=18.0, \Omega=1.50$. This point is just inside the region labeled CHAOS in Fig. 1.

above $\epsilon_{c}$ now lies within the basin of this suddenly created stable attractor and is destroyed in the process, but its dynamic structure persists as a remnant (or ghost). This remnant plays a crucial role in the generation of chaotic transients for this system. The scenario just described is clearly not a boundary crisis. In a boundary crisis a strange attractor approaches the basin of a coexisting stable attractor as a system parameter is varied, and the two make contact when a critical value of the parameter is reached. For the parametrically damped pendulum, the origin is a center of symmetry of both the basin of attraction of the stationary solution and the strange attrac-

(a)

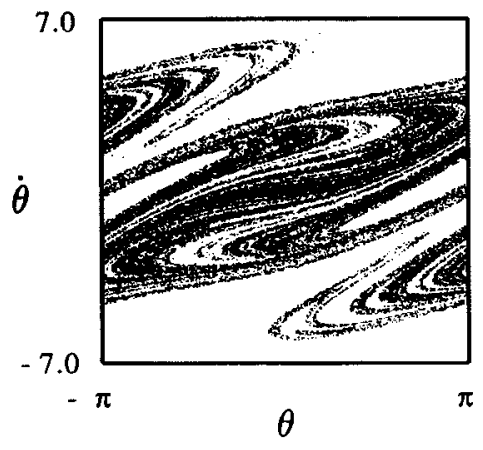

(b)

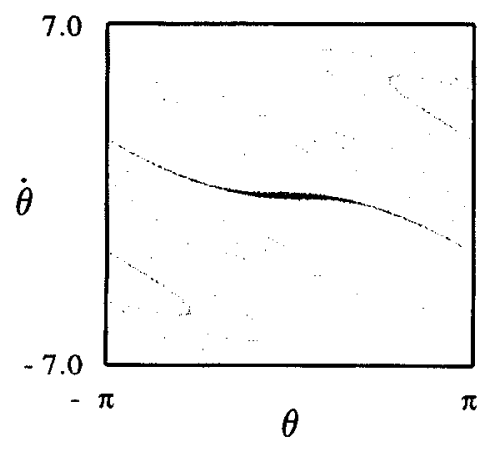

FIG. 4. (a) Strange attractor remnant for $\epsilon=16.0$ and $\Omega=1.50$. (b) Coexisting basin of attraction of the stationary state. tor. This geometric congruence implies either that there is a strange attractor but no stable stationary state $\left(\epsilon>\epsilon_{c}\right)$, or that there is a stable stationary state but only a remnant of a destroyed strange attractor $\left(\epsilon<\epsilon_{c}\right)$. Since the strange attractor and basin never coexist, there is never a collision as such.

The implications of the above remarks may be understood by examining the outcome of integrating equation (2) starting from some initial point selected in the phase plane. A typical trajectory, seen at alternate intervals of one drive period (Poincaré plot), would appear as a sequence of dots moving randomly over the remnant of the strange attractor until, using a phase coined by Grebogi et al. [5], "it finds its way out" and is swept into the attractor at the origin. In other words, the system would exhibit a chaotic transient. Different starting points would lead to quite different trajectories along the strange attractor remnant and would therefore arrive at the stable attractor after different transient times.

From the preceding discussion, it is clear that integrating from a single arbitrarily chosen initial point will yield perhaps only a few points on the strange attractor remnant - before the system is captured by the attractor at the origin. However, by successively choosing many different initial conditions, new points may be accumulated until a composite image emerges. This was the process employed to generate Figs. 4(a), 5(a), and 6(a). In common parlance the strange attractor was "destroyed" (a)

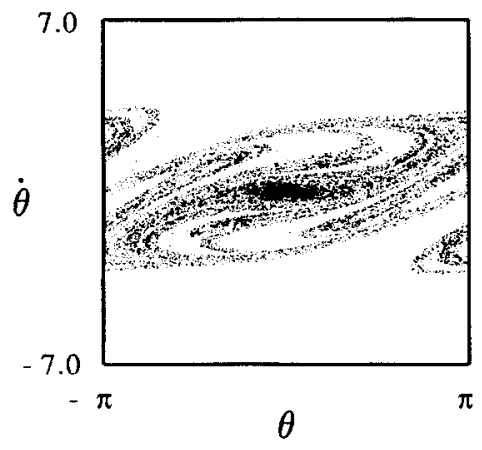

(b)

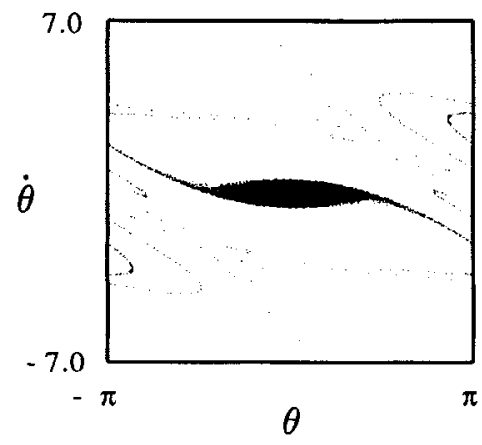

FIG. 5. (a) Strange attractor remnant for $\epsilon=11.0$ and $\Omega=1.50$. (b) Coexisting basin of attraction of the stationary state. 
(a)

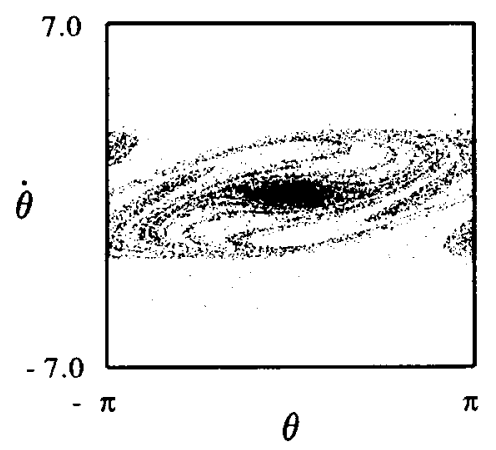

(b)

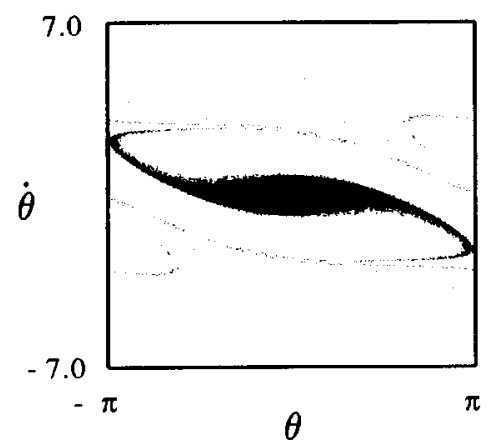

FIG. 6. (a) Strange attractor remnant for $\epsilon=8.0$ and $\Omega=1.50$. (b) Coexisting basin of attraction of the stationary state.

by the event at $\epsilon_{c}$, but it is important to note that the dynamical structure of the strange attractor persists, even far from $\epsilon_{c}$. These figures are images of essentially complete, albeit metastable [17], strange attractors.

To compute a basin for the attractor at the origin, our ICM software routines required the user to specify: (1) the size of the target area surrounding the attractor (2) the number of successive "hits" required within the target, (3) the maximum length of time, in drive periods, allowed for any selected starting point to reach the attractor. Typically, a target dimension of $0.40 \times 0.40$ centered on the origin in phase space was used, and the required number of hits was 10 . The process of choosing a value for the third parameter raises a subtle issue. For $\epsilon<\epsilon_{c}$ chaotic motion is transient, and all trajectories ultimately end in the stationary state. Therefore it could be argued that the basin of attraction consists of the entire phase plane. However, it is a fact that for some starting points the stationary state is reached rather quickly, without any indication of chaos, as if the strange attractor remnant was never encountered. By setting the third parameter to a small value (such as 15 periods), and thus excluding starting points which reach the origin indirectly by first wandering on the strange attractor remnant, the core of the basin of attraction of the stationary state may be acquired. Figures 4(b), 5(b), and 6(b) are a series of such basin cores.

Reviewing the sequence of basins and associated

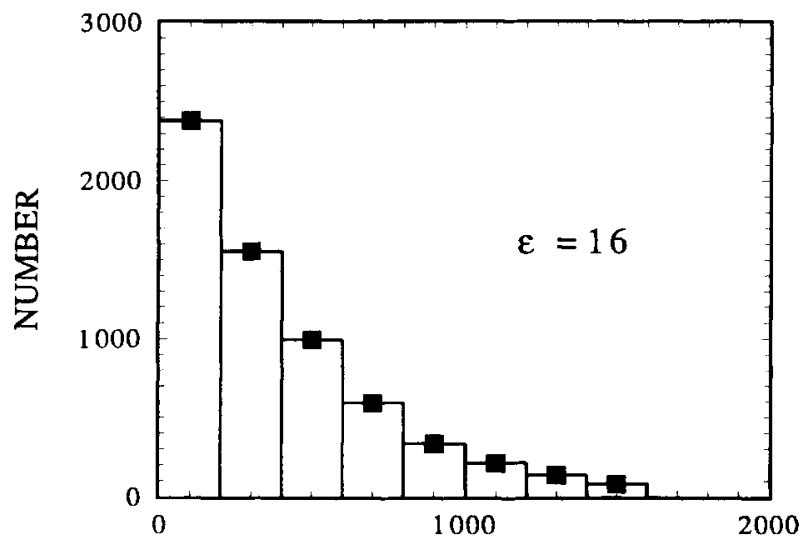

TRANSIENT DURATION (cycles)

FIG. 7. Histogram of grouped data obtained for $\epsilon=16.0$, $\Omega=1.50$, illustrating the relative probability of chaotic transients as a function of the duration of the transient.

strange attractor remnants illustrated in Figs. 4-6, it is evident that as $\left(\epsilon_{c}-\epsilon\right)$ increases, the basin of the stationary solution becomes larger, while the strange attractor remnant shrinks. In combination this means that less time is spent traversing the strange attractor remnant before chaos terminates, and so the mean duration of the chaotic transient will decrease. Ultimately the strange attractor remnant contracts so much that it is completely contained within the basin core, in which case no chaotic transient would be observed.

\section{TRANSIENT CHAOS}

To assess the distribution of chaotic transient times for specified $\epsilon$, and $\Omega$, several thousand starting points were randomly selected from the phase plane, but with the restriction that they not lie on the basin core of the stable solution. For each chosen initial condition, the ICM algorithm was used to determine the time (measured in

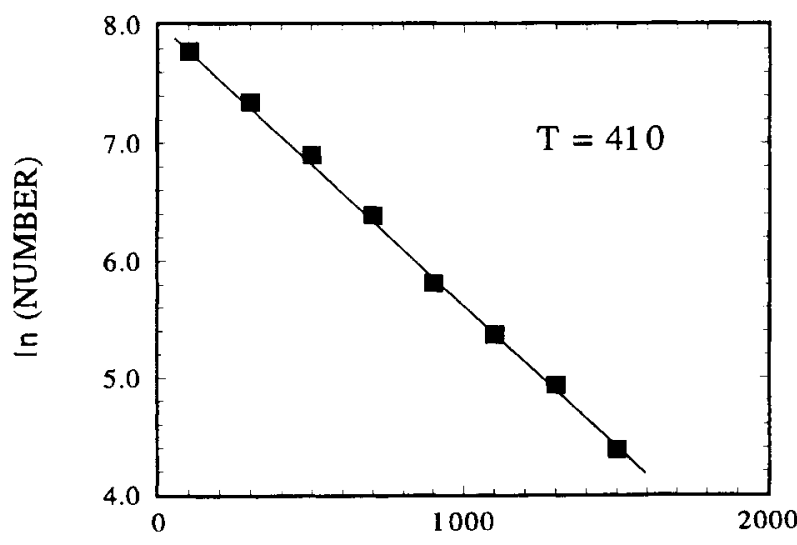

TRANSIENT DURATION (cycles)

FIG. 8. Log plot of data from Fig. 7 and the linear fit yielding the mean transient time $T=410$ cycles. 


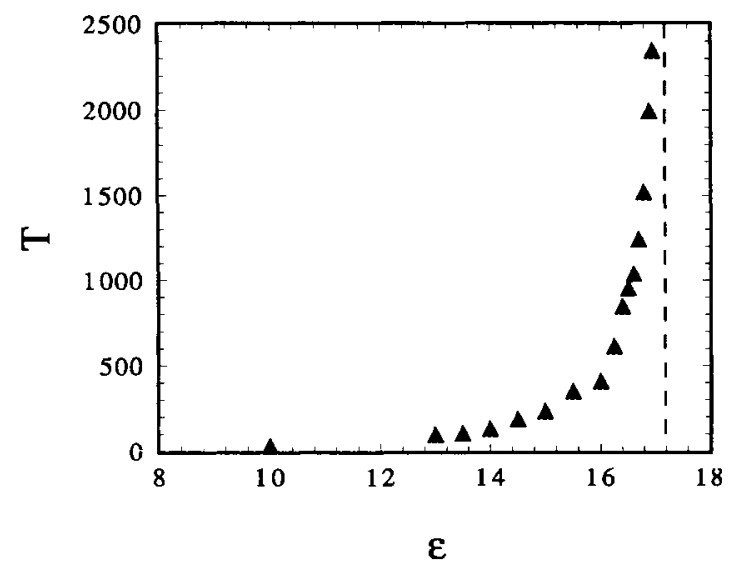

FIG. 9. Mean duration of chaotic transient plotted as a function of the amplitude parameter $\epsilon$ for fixed $\Omega=1.50$. The vertical line indicates the value of $\epsilon$ at the chaotic boundary $\left(\epsilon_{c}=17.2\right)$.

drive cycles) required for the system to evolve to the stable attractor. The accumulated data were then sorted to form a relative probability histogram as shown in Fig. 7 which was computed for $\epsilon=16$ and $\Omega=1.5$. From Grebogi et al. [5], one would expect this distribution to be described by a relationship of the form $P(\tau)$ $=(C / T) \exp (-\tau / T)$, where $P(\tau)$ is the relative probability of observing a chaotic transient of lifetime $\tau, C$ is a constant, and $T$ is the mean transient lifetime. Therefore a plot of $\log _{e}(P)$ vs $\tau$ should be a straight line with slope $-T^{-1}$. The slope of the fitted line in Fig. 8 yields a mean lifetime for the chaotic transient of 410 cycles.

The process described above was repeated for a number of different modulation amplitudes $\epsilon$ (but with fixed $\Omega=1.5$ ). The resulting data are assembled in Fig. 9. As expected, the mean lifetime of the chaotic transient grows rapidly as the boundary at $\epsilon_{c}$ is approached. The log-log plot in Fig. 10 confirms that close to $\epsilon_{c}$ the scaling law is $T \propto\left|\epsilon-\epsilon_{c}\right|^{-\gamma}$ with $\gamma \approx 0.9$. This figure also demonstrates that, even far from $\epsilon_{c}$ where the transient may be

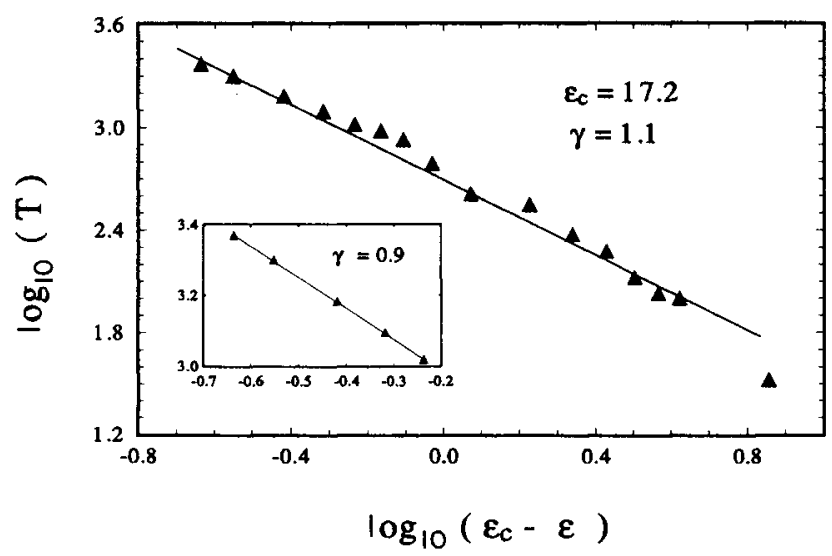

FIG. 10. Log-log plot of data in Fig. 9 showing a scaling relation of the form $T \propto\left|\epsilon-\epsilon_{c}\right|^{-\gamma}$ with $\gamma \approx 1.1$. Close to $\epsilon_{c}$, as shown in the inset, the scaling exponent is 0.9 . very short, a similar power law appears to hold, but with $\gamma \approx 1.1$.

We emphasize the fact that the scenario by which chaos is destroyed at the critical point $\epsilon_{c}$ has not been previously reported to the authors' knowledge. Here there is no boundary crisis. Rather, it is the birth of the new attractor at the origin and the fact that its basin contains the former strange attractor which is the source of chaotic transients. The average duration of these transients depends on the comparative sizes of the basin of the stable state and the persistent metastable strange attractor. For example, small basin cores and large remnants lead to long average transients.

(a)

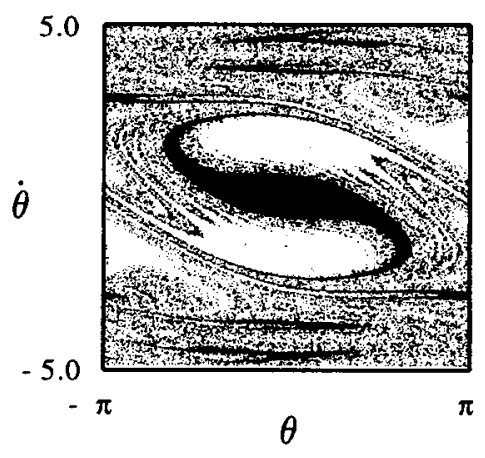

(b)

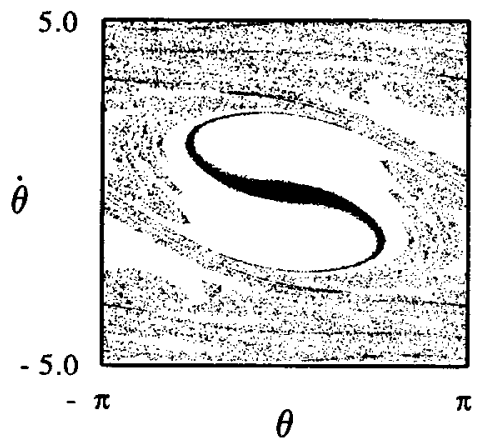

(c)

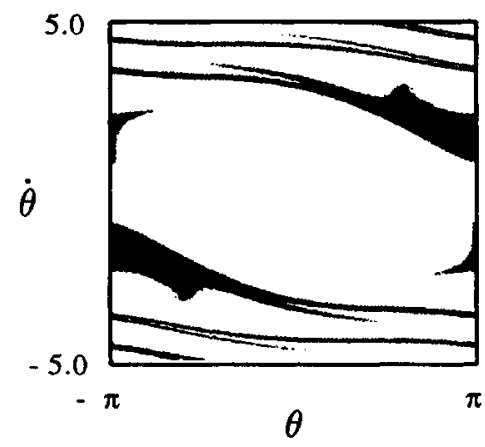

FIG. 11. (a) and (b) Basins of attraction for the stationary state of the pendulum along scan line $B$ in Fig. 1. (c) The stationary state has now disappeared leaving only basins for the spinning states (black) and the oscillating solution (white). 


\section{BASIN TRANSFORMATIONS}

With the aid of interpolated cell mapping, we now examine the changes which occur in basins of attraction for routes labeled $B, C$, and $D$ in Fig. 1. Chaos is not an issue here since all sequences begin in a region where the stationary state is stable, and end in the multiperiodic domain (MP). For route $B$, Figs. 11 (a) and $11(\mathrm{~b})$ illustrate the shrinking basin of the stationary state as the boundary is approached; note that the area lost by this basin is acquired by the double lobed white area, which is the basin for an oscillating solution. At the boundary, the basin disappears as the stationary state becomes unstable, leaving the merged double lobe region as the new basin for the oscillating solution. The black regions in Fig. 11 (c) are basins for spinning states. Comparing them with the matching white regions in Fig. 11(b) reveals that no change has occurred in the basins for the spinning solutions. Crossing the boundary only brings about a shrinkage and the ultimate disappearance of the basin for the stationary state.

Similar patterns are to be found along the routes marked $C$ and $D$ which lead into the multiperiodic domain from the right of the $V$ region in Fig. 1. This boundary was identified in Ref. 16 as a line of Hopf bifurcations. Figure 12(a) illustrates the basins of two coexisting attractors: the stationary state (black), and spinning states (white). As the boundary is crossed into MP along

(a)

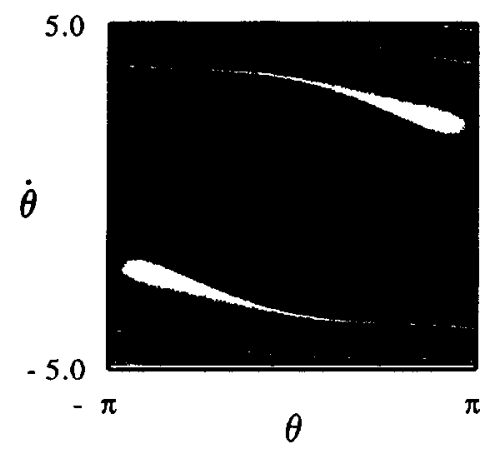

(b)

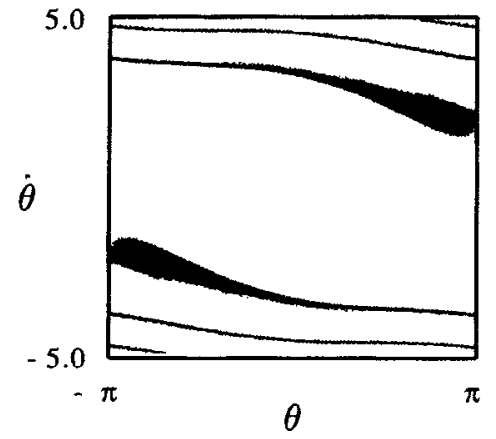

FIG. 12. Basins along scan line C. (a) Basin of stationary state (black). (b) The stationary state has disappeared leaving only basins for the oscillating solution (white) and spinning states (black). scan $C$, the stationary state becomes unstable and its basin spontaneously disappears, only to be immediately converted into a basin for an oscillating solution [white region in Fig. 12(b)]. The basins for the spinning states [white in (a), black in (b)] have not changed at all. A similar sequence develops along scan line $D$. The black region in Fig. 13(a) is the basin of the stable resting state. Again, this abruptly converts to being the basin of an oscillating solution just inside MP, as shown by the white region in Fig. 13(b).

To summarize the results of this section, it has been demonstrated that routes into the multiperiodic domain from across the left-hand boundary in Fig. 1 are accompanied by a shrinkage of the basin of attraction for the stationary solution, and a transfer of the lost "territory" to the basin of the coexisting oscillating solution, until as the boundary is reached, the basin of the stationary solution completely disappears. In contrast, progression into MP across the Hopf boundary on the right is associated with the spontaneous conversion of the entire basin for the stationary state into a basin for the newly created oscillating solution.

\section{ACKNOWLEDGMENTS}

J.A.B. and H.J.T.S. received financial support from the Natural Sciences and Engineering Research Council of Canada.

(a)

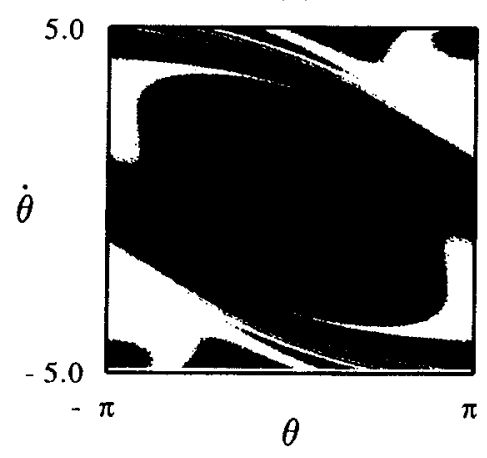

(b)

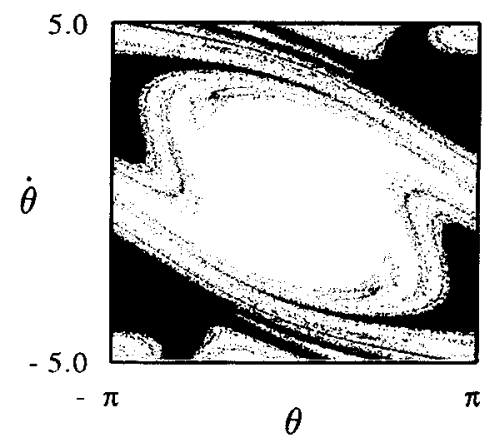

FIG. 13. Basins along scan line $D$. (a) Basin for the stationary state (black). (b) The basin in (a) has been converted to a basin for the oscillating solution (white). 
[1] Y. Pomeau and P. Manneville, Commun. Math. Phys. 74, 189 (1980).

[2] C. Grebogi, E. Ott, and J. A. Yorke, Physica D 7, 181 (1983).

[3] E. G. Gwinn and R. M. Westervelt, Phys. Rev. Lett. 54, 1613 (1985).

[4] W. L. Ditto, S. Rauseo, R. Cawley, C. Grebogi, G.-H. Hsu, E. Kosterlich, E. Ott, H. T. Savage, R. Segnan, M. L. Spano, and J. A. Yorke, Phys. Rev. Lett. 63, 923 (1989).

[5] C. Grebogi, E. Ott, and J. A. Yorke, Phys. Rev. Lett. 57, 1284 (1986).

[6] M. Varghese and J. S. Thorp, Phys. Rev. Lett. 60, 665 (1988).

[7] C. Grebogi, E. Ott, and J. A. Yorke, Physica D 24, 243 (1987).

[8] P. M. Battelino, C. Grebogi, E. Ott, J. A. Yorke, and E.
D. Yorke, Physica D 32, 296 (1988).

[9] C. S. Hsu, J. Appl. Mech. 47, 931 (1980).

[10] C. S. Hsu and R. S. Guttalu, Trans. ASME 47, 940 (1980).

[11] A. Davidson and N. F. Pedersen, Phys. Rev. A 36, 2455 (1987).

[12] A. Davidson and N. F. Pedersen, Physica D 36, 173 (1989).

[13] M. P. Soerensen, A. Davidson, N. F. Pedersen, and S. Pagano, Phys. Rev. A 38, 5384 (1988).

[14] B. H. Tongue, Physica D 28, 401 (1987).

[15] B. H. Tongue and K. Gu, SIAM J. Appl. Math. 48, 1206 (1988).

[16] H. J. T. Smith and J. A. Blackburn, Phys. Rev. A 40, 4708 (1989).

[17] R. L. Kautz, J. Appl. Phys. 62, 198 (1987). 
(a)

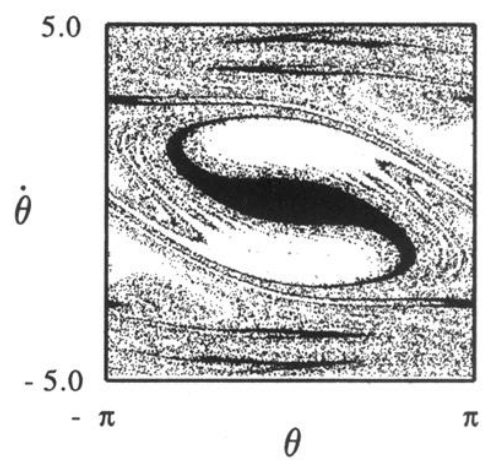

(b)

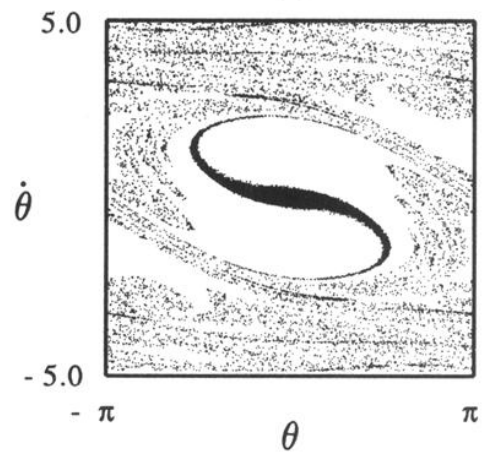

(c)

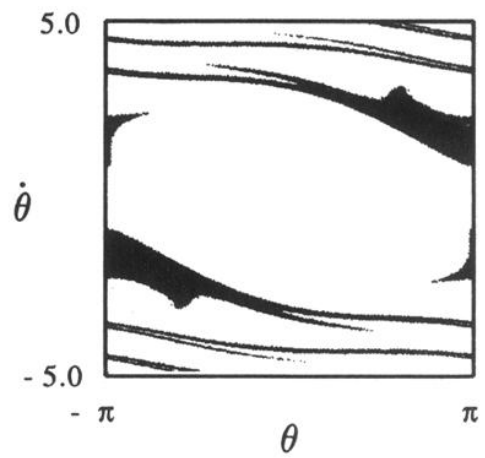

FIG. 11. (a) and (b) Basins of attraction for the stationary state of the pendulum along scan line $B$ in Fig. 1. (c) The stationary state has now disappeared leaving only basins for the spinning states (black) and the oscillating solution (white). 
(a)

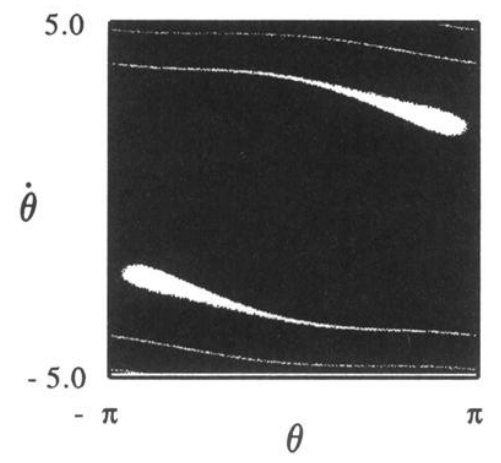

(b)

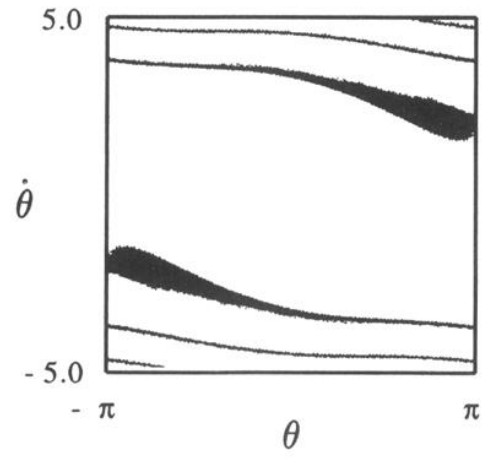

FIG. 12. Basins along scan line $C$. (a) Basin of stationary state (black). (b) The stationary state has disappeared leaving only basins for the oscillating solution (white) and spinning states (black). 
(a)

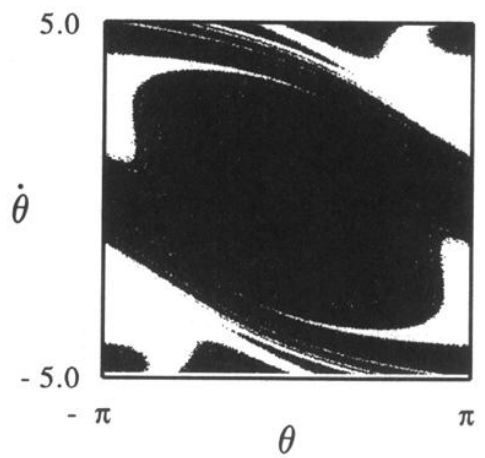

(b)

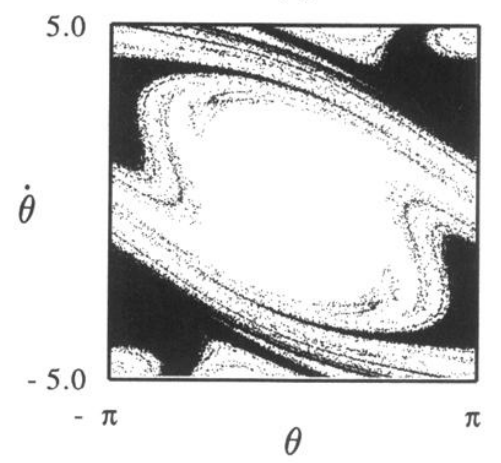

FIG. 13. Basins along scan line $D$. (a) Basin for the stationary state (black). (b) The basin in (a) has been converted to a basin for the oscillating solution (white). 
(a)

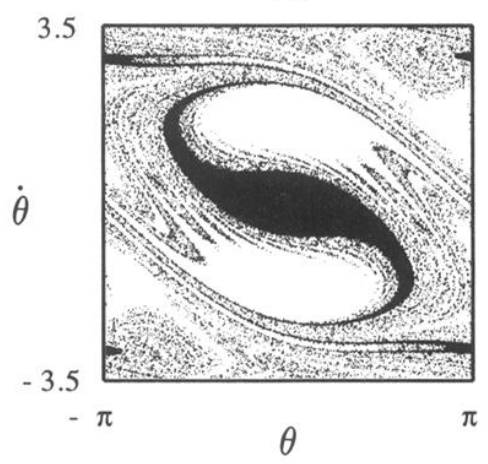

(b)

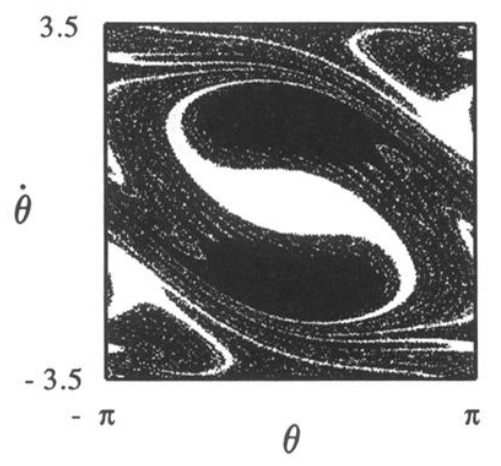

FIG. 2. (a) Basin of attraction (in black) for the stationary solution of the pendulum with $\epsilon=6.0$ and $\Omega=1.70$. (b) Basin of attraction of the oscillating solution. 


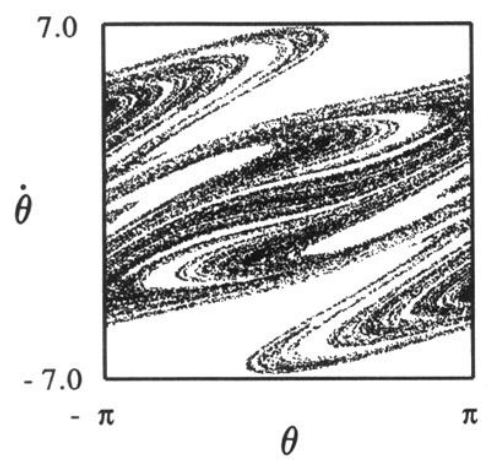

FIG. 3. Strange attractor for $\epsilon=18.0, \Omega=1.50$. This point is just inside the region labeled CHAOS in Fig. 1. 
(a)

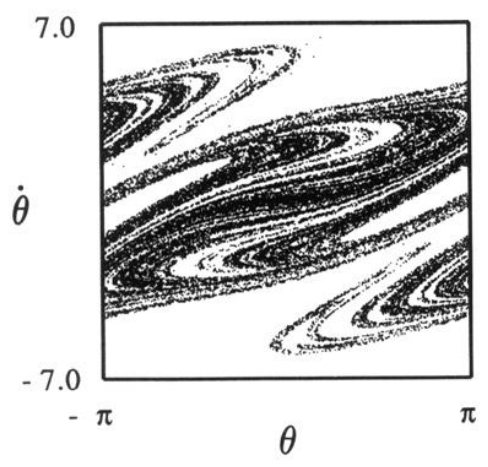

(b)

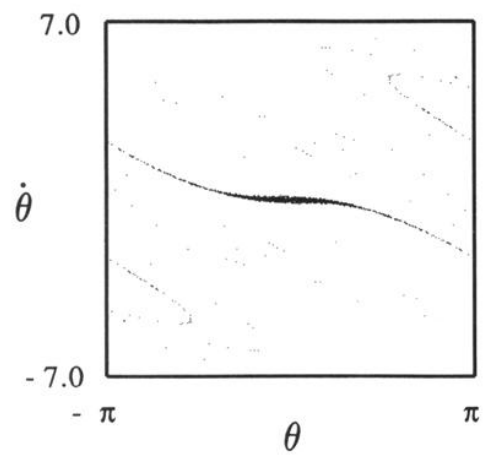

FIG. 4. (a) Strange attractor remnant for $\epsilon=16.0$ and $\Omega=1.50$. (b) Coexisting basin of attraction of the stationary state. 
(a)

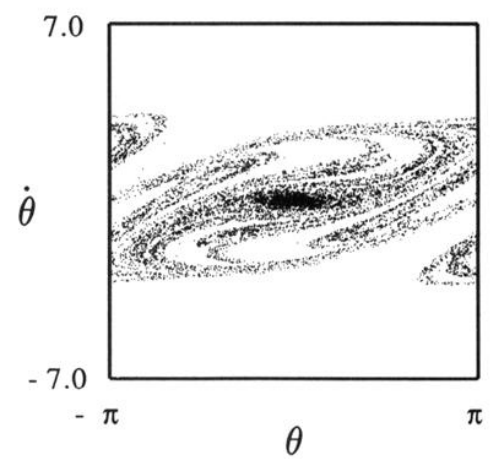

(b)

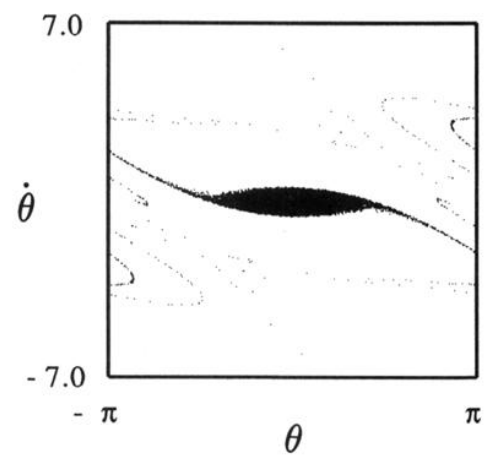

FIG. 5. (a) Strange attractor remnant for $\epsilon=11.0$ and $\Omega=1.50$. (b) Coexisting basin of attraction of the stationary state. 
(a)

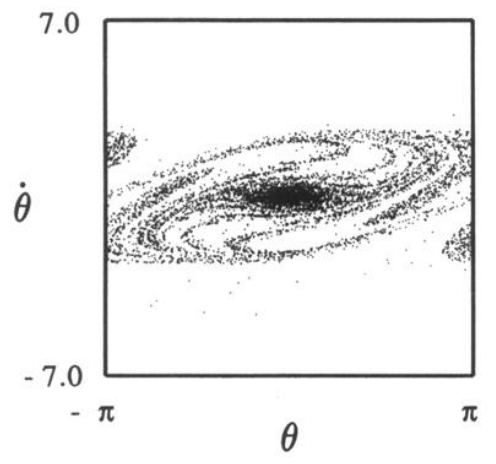

(b)

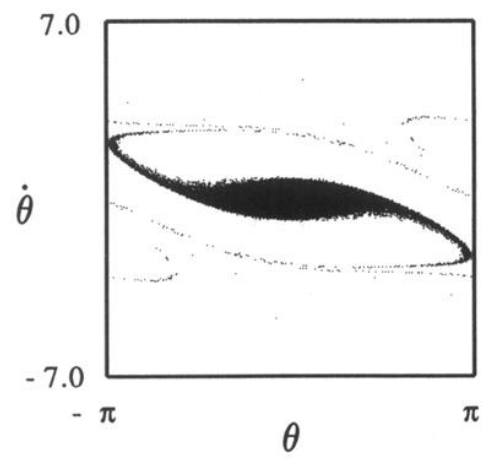

FIG. 6. (a) Strange attractor remnant for $\epsilon=8.0$ and $\Omega=1.50$. (b) Coexisting basin of attraction of the stationary state. 